\title{
Physical principle dealing with development of vortices formed by DBD actuator
}

\author{
P. Procházka ${ }^{1, a}$ and V. Uruba ${ }^{1}$ \\ ${ }^{1}$ Academy of Sciences of the Czech Republic, Institute of Thermomechanics, Dolejškova 1402/5, 18200 Prague, Czech \\ Republic
}

\begin{abstract}
Several configurations of vortex trains generated by plasma dielectric barrier discharge were measured by method of time-resolved PIV with application of phase-averaging of data. Mainly dynamics of vortical structures was studied in terms of vortex core trajectory, convective velocity, vortex diameter and vortex circulation. The generally valid physical mechanism of such vortices based on gained information will be introduced and presented. This mechanism will be considered with regards to the use for active flow control thus it could be very helpful in the future works dealing with plasma flow control.
\end{abstract}

\section{Introduction}

Active flow control by plasma actuators is in the centre of research groups from the nineties of the last century. These unconventional devices are used for their advantages in comparison with other active control methods as synthetic jets. The laminar-to-turbulent transition, the flow separation or turbulence itself can be influenced by plasma actuation. Nevertheless, the effect and particularly the efficiency remain rather small. The applicability for industrial cases is very limited because the Reynolds number of majority technical problems exceeds the value of $10^{3}$. Instead the research was focused on better understanding of the plasma actuators function resulting in an increase of the efficiency or at least more sophisticated active control at the capabilities of contemporary devices, there is presented the influence of plasma-induced thrust on small-scale applications as boundary layer of MAV wing.

Previous research showed [1] that plasma actuators in unsteady regime are able to achieve improvements of controllability and effectiveness by the reduction of power consumption. This unstable regime and its careful study may therefore be the key to improve the efficiency. The phenomena (vortex structures) that occur in unsteady regime have strong dynamic character. This article deals with the study of series of vortices generated by DBD (dielectric barrier discharge) actuator, the description of dynamics and specification of generally valid physical model that can predict the vortex development.

The experimental investigation of flow field around DBD actuator is a great challenge for PIV and other velocity measure technique. The gained information about 2D field can serve to fulfill boundary conditions of CFD simulation and more can be implemented by plasma actuation in the future.

\section{Experimental layout}

The plasma actuator is the same as in the previous study [2]. It is a simple DBD plasma actuator whose one electrode is very thin wire $(40 \mu \mathrm{m})$ and is powered by high-voltage high-frequency waveform. The second electrode is grounded and both electrodes are separated by silica glass dielectric. In case of unsteady actuation, there is applied amplitude modulation on voltage waveform resulting in series of vortices appearing in plasma-induced wall jet.

The power source has so-called shut down function which allows incorporate amplitude modulation. Then four electric parameters come into account. The peak voltage magnitude of waveform and the waveform frequency can be set by this power source, but they are tuned constantly (approximately $16 \mathrm{kHz}, 11 \mathrm{kV}$ ) during entire measurement as it can generate most powerful electric wind (wall jet). The other two parameters frequency of modulation and duty cycle (DC) are to be fully adjustable during measurement and are determined to define the characteristics of "vortex train". The order of modulation frequencies is tens of $\mathrm{Hz}$, because the higher frequencies are used, the vortex train is more similar to steady (unmodulated) case. Duty cycle determine the rate between the working cycle of actuator (plasma is on) and relaxation time (plasma is off).

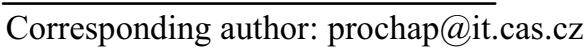


Measured frequencies were 5, 10, 15, 20, 25, 30, 35, 40, $50,60,70,80$ and $90 \mathrm{~Hz}$ with duty cycle of 30,50 and 70 $\%$. Detailed examination of duty cycle (after $10 \%$ ) was performed on the frequency $20 \mathrm{~Hz}$. Finally, the general physical model was created at the bases of these configurations.

The plasma actuator was placed inside a plexi-glass chamber to prevent the adverse influence of ambient air in the laboratory to plasma-induced wall jet. Then the actuator formed the bottom of the chamber. The velocity measurement in the vicinity of the actuator was performed by TR-PIV (detailed technical data see in [2]) and by hot wire anemometry (see [3]). Since the phenomenon of unsteady actuation generate coherent vortical structures, that have pseudo-periodical character, phase-averaging of data were used by data acquisition. Then the random components (e.g. random fluctuation of the vortex core) were filtered out thus the periodic behaviour could be sophisticatedly observed and described.

Data acquisition and evaluation of velocity vector map was performed in DynamicStudio software. Phaseaveraging, vortex identification and further computation were performed in Matlab. The Labview software was used to ensure data acquisition and processing in the case of HW anemometry when only frequencies present in the wall jet were taken into account.

\section{Results}

It can be seen how the wall-jet-like flow looks like at figure 1. This type of steady flow was studied previously [4] and here it will be mentioned only to remind. The continuous wall jet extends from wire powered electrode in a horizontal direction toward the place where lower electrode is located. Maximum peak velocities present in the jet reach the value of $3 \mathrm{~m} \cdot \mathrm{s}^{-1}$. Note, that direction of electric wind is from right side to the left, unlike general ingrained habits. The reason is strictly constructional.

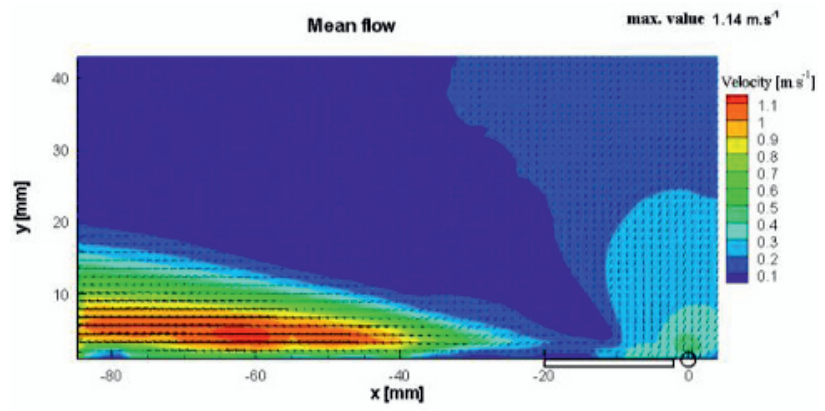

Figure 1. Basecase - steady regime, unmodulated flow field

The incorporation of modulation into voltage waveform results in the existence of period vortex structures in the plasma-induced electric wind. The figure 2 shows an example of that kind of flow where the modulation frequency is $35 \mathrm{~Hz}$ and $\mathrm{DC}$ is $50 \%$. The coordinate origin denotes the position of powered wire electrode. The vortex detection is performed using vorticity distribution, in the process the blue area denoted the location of vortex core and red area are associated only with shear layers (the vortices demonstrate only clockwise rotation). Already from this figure it is clear that the size of vortical structures and their mutual distance will be a function of driven parameter of amplitude modulation - the frequency and duty cycle. At the end of this article, a relation that outlines these dependencies on the basis of gained data will be presented.

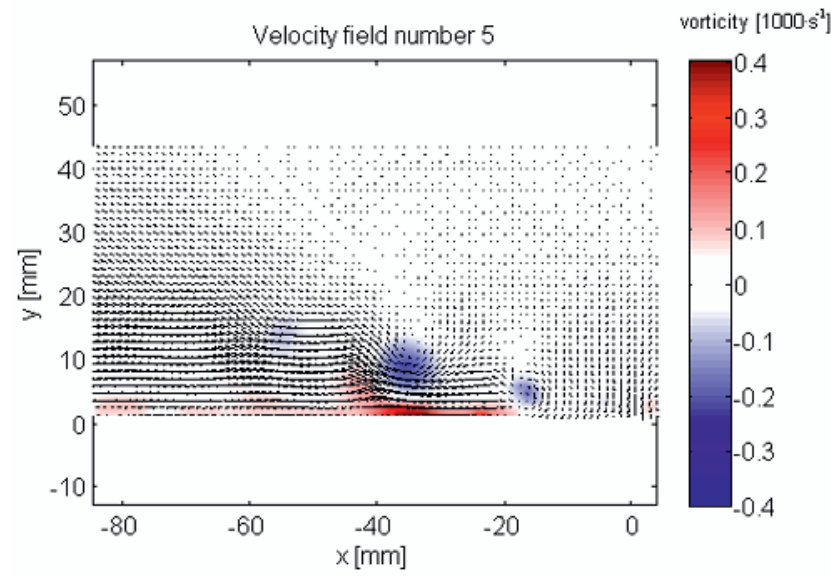

Figure 2. Unsteady regime - Modulated by $35 \mathrm{~Hz}$ and $50 \%$

The Reynolds number of this kind of flow must be defined slightly unconventional because there is no inlet velocity and no orifice diameter, so the Reynolds number is defined as

$$
R e=\frac{U_{\text {localmax }} \cdot \delta}{v}
$$

to be able to quantitatively describe the flow. The numerator is a product of the maximum velocity present in the velocity profile far from wire electrode and thickness of the jet in the same place. The boundary of the plasma jet is defined as existence of $50 \%$ of the maximum velocity value in the velocity profile at the same place. The Reynolds number depends on both the velocity magnitude and the jet thickness. Since these two value go against each other, it is not desirable to follow only absolute value of $\mathrm{Re}$, but each individual increments of individual parameters when comparing Re number of different configurations. The Reynolds number develops along the electric wind propagation (See figure 3 ).

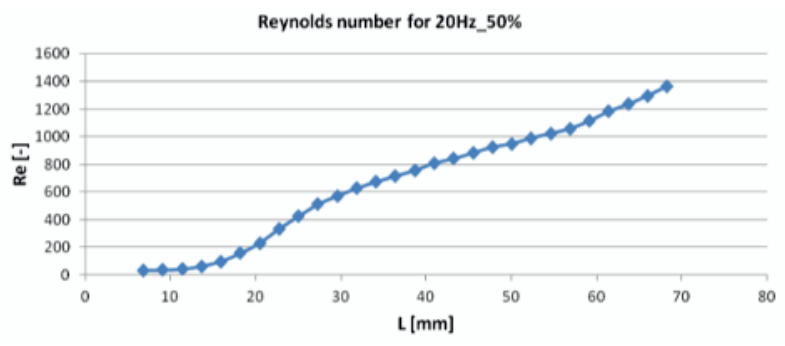

Figure 3. Reynolds number development along streamwise direction 


\subsection{Vortex dynamics}

The dynamical behaviour of vortex train should be presented here on the example of one vortex life. The vortex is created immediately after the start of working cycle. The vortex is fed by the body force energy during the working cycle and is blown away from wire electrode, thus the effect of electric wind on the vortex becomes weaker. There is no more electric wind during relaxation time and vortex structures develops individually which results in vortex breakdown every time. For lower frequencies, the vortex breaks down before the working cycle ends, so then there is only the wall jet flow disturbed by velocity components of previously perished structure until the working cycle ends.

The description of dynamics will be performed by convective velocity of the vortex core (evaluated from vortex core trajectory), the equivalent radius and the circulation. Based on that, the physical model dealing with vortex creation and development will be presented in discussion.

The vortex core was identified for every phase of one vortex period by identification schemes based on velocity gradient tensor. Then vortex core trajectory was reconstructed for every configuration by a regression curve of the second order (there are some limitation in spatial resolution due to PIV technique resolution) [5]. The convective velocity is almost constant for whole period and is computed as an average of individual increment of trajectory and depicted for every configurations at figures $4 a, b$.

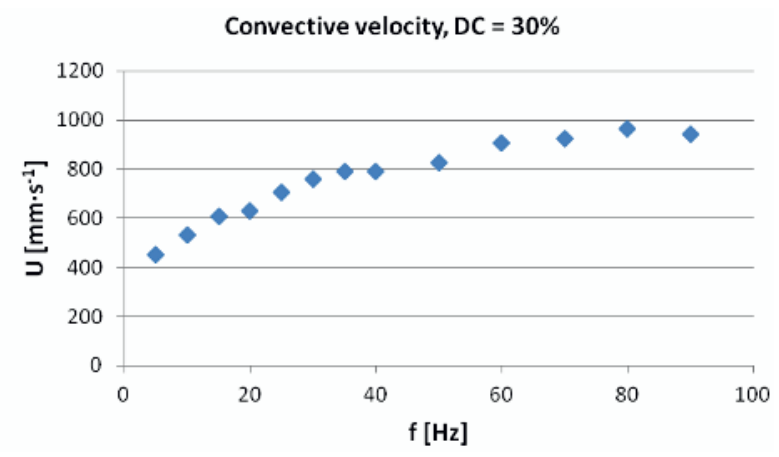

Figure 4a. Convective velocity of the vortex core versus modulation frequency

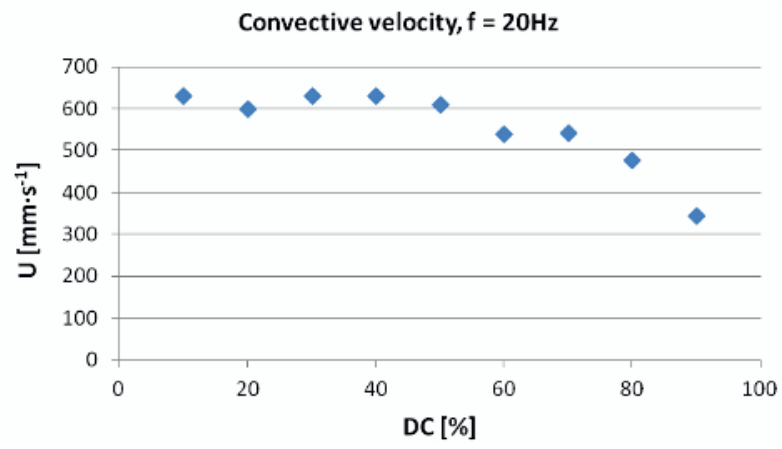

Figure 4b. Convective velocity of the vortex core versus duty cycle
The convective velocity significantly differs from the velocity of electric wind and more significantly from the self-induced velocity of each vortex. The explanation will be given in discussion. Convective velocity is increasing with applied frequencies of modulation and is decreasing with duty cycle.

The equivalent radius is defined as

$$
R_{e e q v}=\sqrt{\frac{2 S}{\pi}}
$$

where $\mathrm{S}$ is surface of only upper part of vortex. The boundary of vortex is considered as an existence of $10 \%$ of extreme scalar value of the core. This threshold value is determined for each phase of vortex period separately. This fact must be taken into account when comparing two different configurations. The radius of vortex is increasing for all configurations in time (per phases) and the biggest value occurs for the lowest frequencies. The dependency on duty cycle is rather unclear (figure $5 b$ ), and it seems that the definition (2) is not convenient for this purpose.

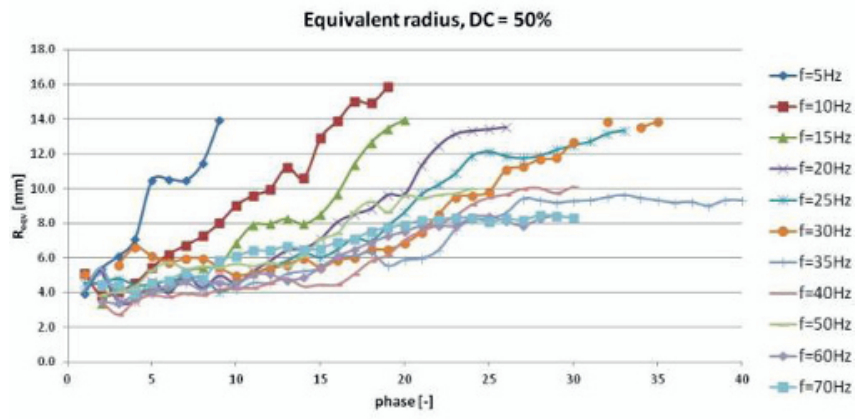

Figure 5a. Equivalent radius for $\mathrm{DC}=50 \%$

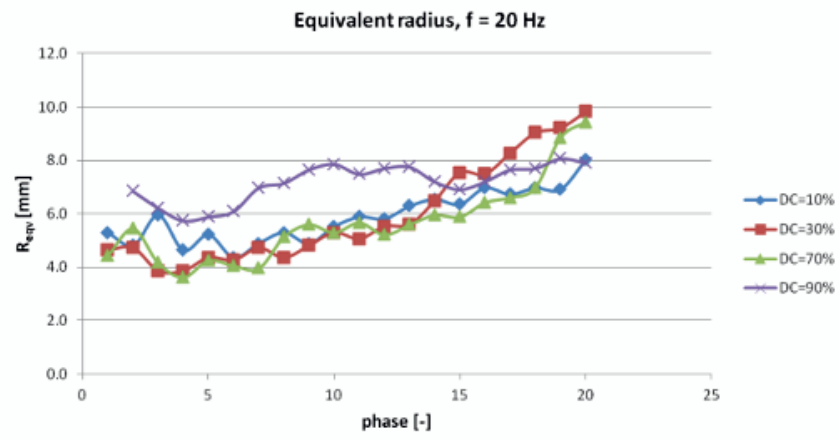

Figure 5b. Equivalent radius for $\mathrm{f}=20 \mathrm{~Hz}$

The most important feature is the circulation of the vortex in terms of the dynamics investigation. The circulation is not affected by a significant error due to integration the values of circulation in the area under interest. Then the accuracy of that value reaches $0.1 \%$. The figure $6 \mathrm{a}$ denotes the circulation evolution in time for vortex structures generated under various frequencies and driven by $50 \%$ of duty cycle. Since the duty cycle is identical for all configurations, the dotted line marks the end of the working cycle - when the electric wind is turned off. The circulation is rising almost as a quadratic function during all working cycle and even still after the 
end. This implies that there is some inertia in energy feeding of the vortex. The duration time of circulation increasing is almost identical for every configurations (increasing number of phases). There is a rapid fall of vorticity or slowly decreasing until the vortex breakdown occurs for lower frequencies and form higher frequencies, respectively.

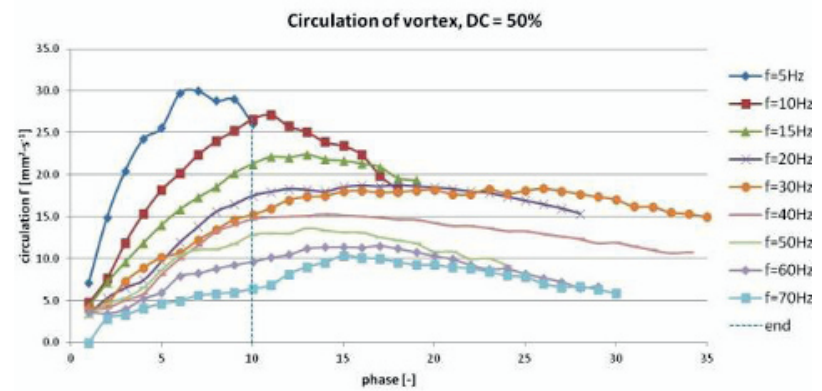

Figure 6a. The circulation development in dependency on frequency

The figure $6 \mathrm{~b}$ denotes the situation when the changing parameter is duty cycle (modulation frequency is fixed at $20 \mathrm{~Hz}$ ). It can be seen that duty cycle has strong dependency on vortex massiveness when a higher duty cycle does not enable to vortex to gain maximum achievable circulation given by the particular configuration. However, the circulation accounts the same behaviour up to the end of working cycle of highest duty cycle when the circulation rises here almost linearly. The development after the end of working cycle proves similar behaviour as in the figure 6a. There is a slow decline or rapid fall for higher duty cycle or for lower duty cycle, respectively.

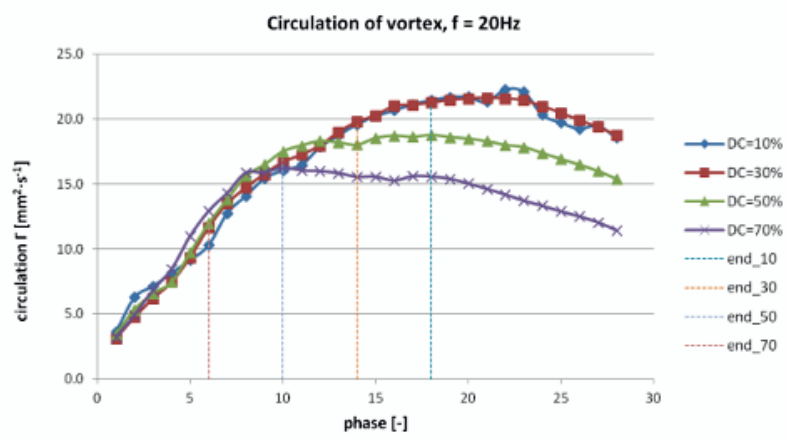

Figure 6b. The circulation development in dependency on duty cycle

The duration of vortex life differs for every configuration. The longest time occurs for the lowest frequencies that can generate most powerful circulation which can help the vortex to resist. More, it is valid that the time of vortex life is slightly longer for DC of $30 \%$.

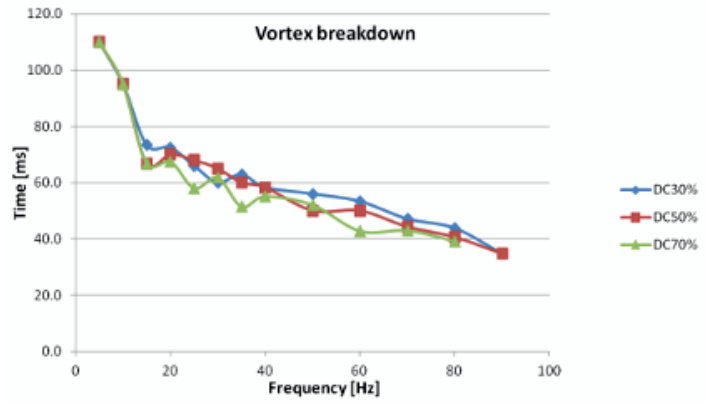

Figure 7. Vortex life duration

\subsection{Frequency spectrum}

The main task of frequency analysis (based on HW anemometry data) is to determine the high frequency that could be present in the flow and that are out of temporal resolution of TR-PIV. The power source generates unfortunately very strong electromagnetic radiation which influences the probe itself. The background signal of HW probe (when it was not exposed to the electric wind) was taken (figure 8) where can be clearly seen the peak frequency value of $16 \mathrm{kHz}$ which corresponds to the frequency of power source voltage waveform. The difference between effective signal and the background of the probe is comparable order of magnitude. It cannot be said if there are some high frequencies present in the wall jet.

Finally, the biggest interest is about the lower frequencies that are incorporate by amplitude modulation. Here, the effective signal is larger by several orders as compared with the background in this area. From power spectral density (SPD) diagram can be seen that the modulation frequency and higher harmonics are dominant (see figure 9). This topic is detailed presented in [3].

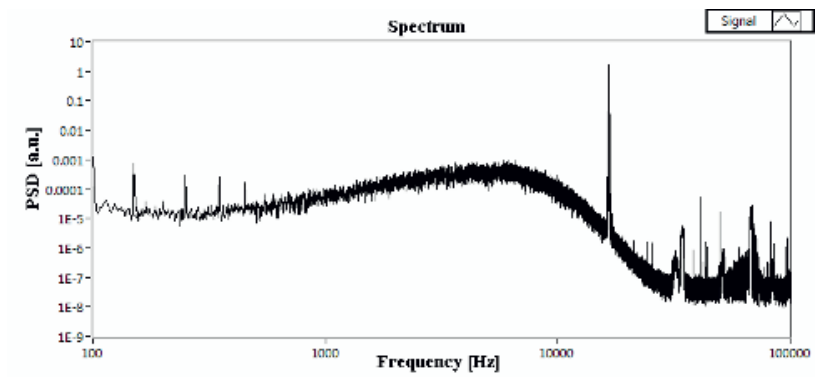

Figure 8. The background signal of HW probe

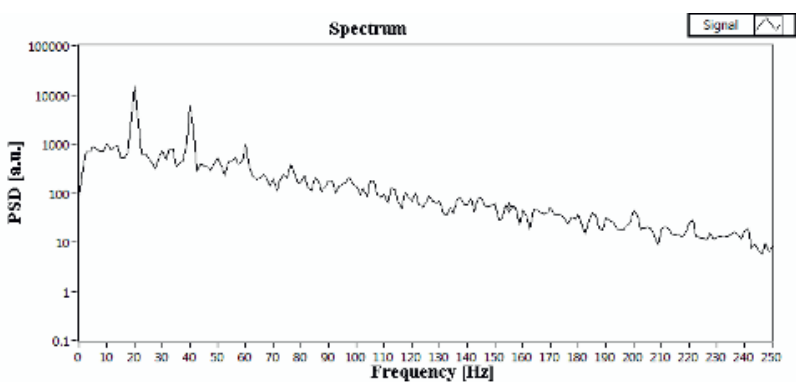

Figure 9. Frequency spectrum of modulated case $-20 \mathrm{~Hz}$ and $50 \%$ 


\subsection{Discussion of results}

The plasma jet is very tightly adhered to the surface throughout of its existence (meant for steady case). This is surely cause by Coanda effect. The flow has tendency to be attracted to a nearby surface. This is a result of an entrainment of ambient fluid around the wall jet. The solid surface does not allow the surrounding fluid to be pulled inwards towards the jet and the jet moves toward the wall instead. This property seems to be very convenient concerning the active flow control applications.

The reasons why the modulated electric wind can generate vortical structures are depicted in [5]. Now let us look how the convective velocity of the vortex is related with Biot-Savart law:

$$
u_{\text {ind }}=\frac{\Gamma}{2 \pi r}
$$

The equation (3) is valid for infinity vortex line, where the induced velocity is computed in some point far from vortex line in $r$ distance. Induced velocity influence the vortex movement both in horizontal sense and in vertical sense. When the vortex is close to the surface, the method of the image vortex is used. The imaginary vortex is projected behind the solid surface and the distance $r$ is considered as a double. Since the circulation value and the vortex distance from the wall is known for all configuration per time, the value of self-induced velocity can be computed and compared with current known convective velocity of vortex core.

Although the circulation and the distance from the wall is varying in time, the self-convective velocity is almost constant (as was measured). But the computed induced velocity of vortex is orderly smaller than measured convective velocity which is a clear evidence that electric wind is a originator of vortical structures and more electric wind acts on the bottom side of the vortex and thus it gives a momentum which remains still the same along the investigation frame of interest. The dependency of convective velocity is given by only modulation frequency and duty cycle and can be determined from sampled data as a quadratic equation [5].

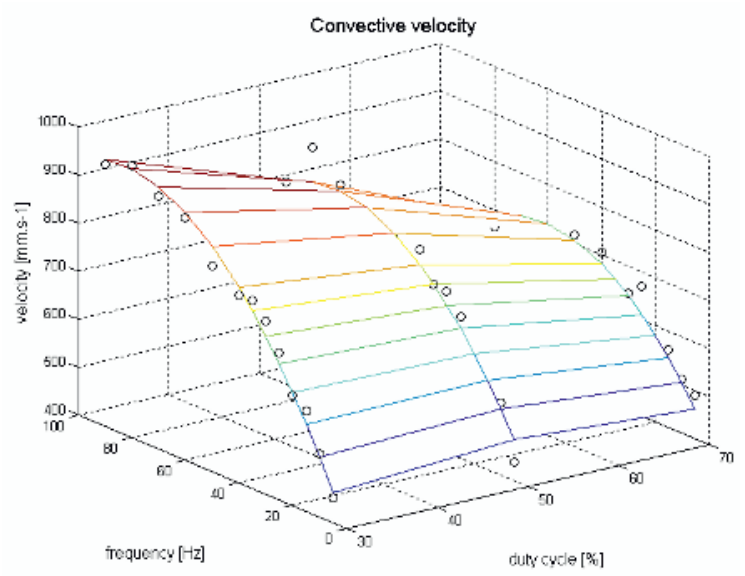

Figure 10. Measured convective velocity of the vortex core and regression fitting of the second order
The current graph at the figure $6 \mathrm{a}$ has to be modified, if the gradient of circulation increase in time is desirable. This adaptation would cause that the circulation is plotted on dependency on time in seconds. Now it would be easy to see that the gradient of the circulation increase is almost identical for all configurations (with except of high frequencies).

The vortex breakdown occurs not from the reason of energy dissipation. The initial vortex filament is twodimensional but it starts to change its orientation in space and three-dimensional instabilities are going to destroy it.

\section{Conclusions}

Several configuration of amplitude modulation resulted in series of vortices in plasma-induced electric wind. The flow field was measured by TR-PIV and HW anemometry and data were consequently evaluated in Matlab. Vortex core identification was performed and each configuration of modulation was quantitatively described by quantities expressing the dynamical nature of that phenomenon. These quantities were especially convective (drift) velocity, equivalent radius and circulation of the vortex. Based on gained data, a generalization of unsteady regime of DBD plasma actuator was performed.

The DBD actuator demonstrated the ability to generate the desirable vortical structures according to input power parameter. The most important and promising is the fact that sufficient strong electric wind was generated without or with the presence of vortices that can be driven which can be used to flow control. There is no doubt that this device can be used for active flow control with steady or unsteady actuation after a cosmetic design adaptation.

\section{Acknowledgement}

The authors gratefully acknowledge financial support of the Grant Agency of the Czech Republic, No. 101/08/1112.

\section{References}

1. E. Moreau, Journal of Physics D: Applied Physics 40, 605-636 (2007)

2. P. Procházka, V. Uruba, Experimental Fluid Mechanics, 594-598 (2012)

3. P. Procházka, V. Uruba, P. Antoš, XXXII Setkání Kateder Mechaniky Tekutin a Termomechaniky, 243246 (2013)

4. P. Procházka, V. Uruba, Proceedings in Applied Mathematics and Mechanics, 1, 667-668 (2011)

5. P. Procházka, Vortex structures generated by plasma DBD actuator, Dissertation thesis, ČVUT (2013) 